\title{
Artificial Muscle for Small Robots and Other Micromechanical Devices
}

\author{
Non-member Ron Pelrine (SRI International) \\ Non-member Roy Kornbluh (SRI International) \\ Member Seiki Chiba (SRI International)
}

\begin{abstract}
This paper discusses a fundamental research project aimed at developing. The project was motivated by the need for better microactuator technology that can be used for micro-robots and other micro applications. A range of artificial muscle technologies was investigated, and a class of electroactive polymers based on the electric field-activated deformation of dielectric elastomers was selected as the most promising approach for an in-depth-study. The dielectric elastomer polymers are sandwiched between two compliant electrodes. Actuation is accomplished by the application of a voltage difference between the two electrodes, which squeezes and stretches the film. A variety of polymer and electrode materials have been tested. Excellent actuator performance has been achieved by this approach, with actuated strains over $300 \%$, actuation pressures of 1-8 MPa, response times of less than $0.5 \mathrm{~ms}$, and theoretical efficiencies of over $80 \%$. Corresponding specific energy densities exceeded $3 \mathrm{~J} / \mathrm{g}$, and are believed to be the highest ever observed for a field-actuated material, based on the available literature..
\end{abstract}

Keywords: electroactive polymers, artificial muscle, actuators

\section{Introduction}

Micro-robots and many other microelectromechanical systems (MEMS) require high performance actuation. This need is especially acute at small scales, because conventional electromagnetic motors generally have poor performance when they are reduced to micro dimensions. Because of this need, SRI International undertook an investigation of micro actuator technology under the sponsorship of the Micro Machine Center. The project was named "Artificial Muscle for Small Robots" to indicate that the investigation would focus on muscle-like actuation.

Muscle-like actuation has several distinct advantages over existing actuation technology, at both micro and macro scales. Natural muscles are distinguished by their excellent overall performance, as measured by several performance parameters (strain, pressure, speed, etc.) rather than just one. Figure 1 shows stress and strain performance of muscles found in several invertebrate species. In contrast to natural muscle, most conventional actuators excel in one or two measures of performance but are poor in others. For example, piezoelectric materials have excellent pressure performance but poor strain. Shape memory alloy has excellent energy density and reasonable strains but poor efficiency and slow speeds. Electromagnetics have good speed and displacement but relatively low pressures and poor efficiency at slow speeds. Besides its excellent overall performance, natural muscle is distinguished by its scale invariance: thus, the muscle of large creatures such as whales and elephants is similar to that of small creatures such as mice and insects.

An artificial muscle that could mimic the performance of natural muscle could have a major impact on actuator applications. If its performance is truly comparable to that of natural muscle, its high strains and high energy density would eliminate the need for complex transmissions (such as gearing) in many applications. Its scale invariance enables it to be applied at both micro and macro scales, and its high-speed capabilities make it ideal for a wide range of micro applications such as micro-robots, inkjets and other micropumps, and MEMS optical elements.
In 1992 SRI International (SRI) began an investigation of a wide range of artificial muscle approaches that might be applied at micro scales (1). Technical goals were set for muscle-like performance. The final goals were $45 \%$ strain; $0.5 \mathrm{kPa}-\mathrm{m}^{3} / \mathrm{kg}$ actuation pressure-density ratio (a measure of force-to-weight characteristics); $80 \%$ theoretical efficiency; and $0.5 \mathrm{~ms}$ response time. These goals compare favorably with the performance of natural muscle, and, if met, would represent a significant advance in actuator materials.

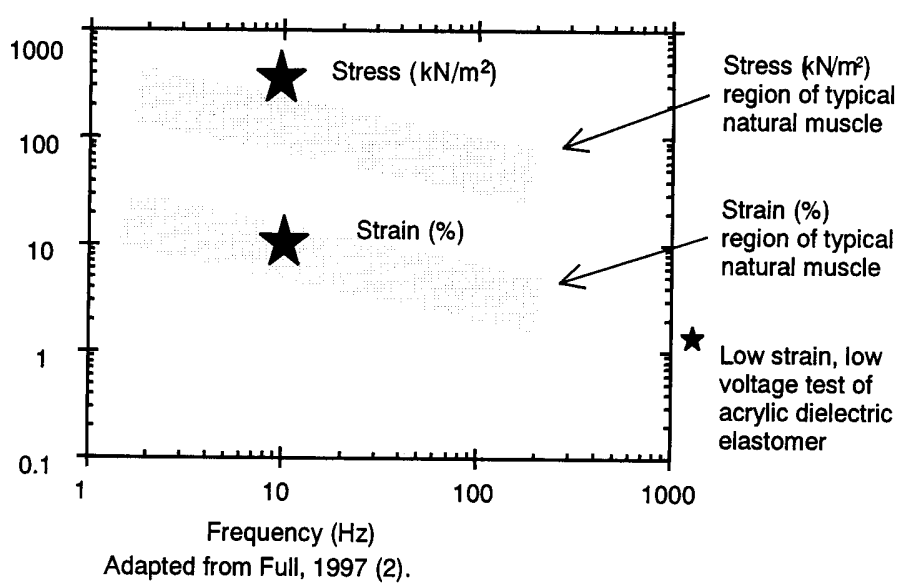

Fig. 1. Low-strain, low-voltage test of acrylic elastomer compared with various types of natural muscle.

In the early stages of our investigation we evaluated a number of existing actuator technologies such as electromagnetics, piezoelectrics, electrostatics, shape memory alloy, and shape memory polymers for application to artificial muscle. Unfortunately, existing actuator technologies appeared unable to meet the goals of an artificial muscle, even if developed specifically for this purpose. For this reason, we explored and evaluated several novel actuation approaches. 
One new approach appeared particularly promising, though little was known about it initially. It was, however, well known that certain polymers such as PVDF could respond as piezoelectric materials. At the early stages of the project, electrostrictive polymers such as polyurethane (3) were under investigation and showed better response than that of piezoelectric polymers. However, both piezoelectric and electrostrictive polymers had strains too low to achieve the project goals.

Polymers, and indeed all insulating materials, also respond electromechanically by means of a third actuation mechanism known as Maxwell stress. In essence, Maxwell stress is caused by forces generated electrostatically when electrodes apply voltage across an insulating material. Previously, Maxwell stress was regarded as a negligible effect; most published articles on piezoelectric and electrostrictive materials carefully eliminated it as a source of measurement error to show that piezoelectric or electrostrictive effects were present.

The mechanism of Maxwell stress in a polymer film is shown in Figure 2. Compliant electrodes are placed on the top and bottom surfaces of the film. When a voltage difference is applied between the electrodes, the electrostatic force squeezes the film in thickness and stretches it in area.

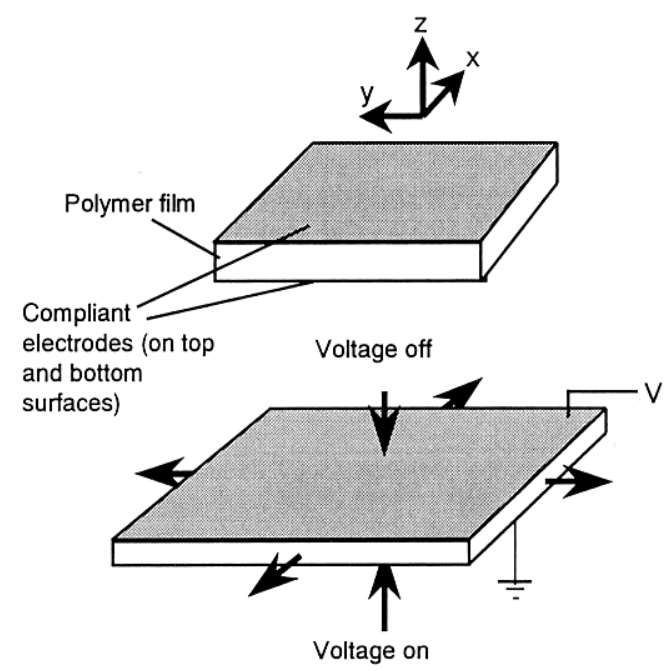

Fig. 2. Principle of operation.

As part of our evaluation of actuator technologies, we tested polyurethane films that were reported to have a large electrostrictive response. We did not observe significant electrostrictive response in our initial polyurethane tests, but we did observe high breakdown strengths in thin polyurethane films. From this observation we reasoned that a Maxwell stress mode of operation using elastomers might yield muscle-like performance. Furthermore, other elastomers have as good as or better dielectric properties than those of polyurethane, so that other elastomers might yield superior actuation response. This observation was critical because the electrostatic response is shared by all elastomers; on the other hand, the purely electrostrictive response of polyurethane is restricted to a limited class of semicrystalline elastomers. Thus, by focusing on the electrostatic response, we can apply this approach to a wide range of polymer materials.
This novel actuation method based on the use of Maxwell stress to actuate polymers has yielded the promising results described below. These polymer materials are also sometimes referred to as electrostrictive, in the sense that their response is proportional to the square of the field (in contrast to piezoelectrics, where the response is linear in the field). However, the term electrostrictive is often reserved in the literature to describe materials that respond to molecular changes and/or charge injection into the film. For this reason, we will refer to polymers whose major response to an electric field is due to Maxwell stress as dielectric elastomers, to emphasize that for this type of material it is the bulk dielectric properties that are important.

\section{Theory}

The use of Maxwell stress as an actuation mechanism has been studied theoretically, and analysis tools have been developed to better understand the resulting behaviors. The fundamental mechanism can be analyzed by means of electrostatic theory in a polymer dielectric medium of dielectric constant $\varepsilon$. The effective actuation pressure, $p$, exerted on the film is given by

$$
p=\varepsilon \varepsilon_{\mathrm{o}} E^{2}
$$

where $\varepsilon_{0}$ is the permittivity of free space $\left(8.85 \times 10^{-12} \mathrm{~F} / \mathrm{m}\right)$ and $E$ is the electric field in $\mathrm{V} / \mathrm{m}(4),(5)$.

Two observations made from Equation 1 clarify the differences between Maxwell stress actuation and the use of conventional air-gap electrostatic actuators. First, $\varepsilon$ for polymers is typically in the range $2-12$, whereas for air $\varepsilon$ is 1 . Thus, the actuation pressure is increased substantially via polymers rather than air at the same electric field. Another difference is that typical air-gap actuators have an additional factor of 0.5 in their equivalent pressure expression, i.e., the polymers double the actuation pressure independent of the dielectric constant. The reason for this difference is that the polymers can stretch in area rather than just contract in thickness. Polymers have two modes of converting electrical to mechanical energy. In contrast to polymers, air-gap actuators are typically made of rigid materials that can convert electrical to mechanical energy via only one mode of motion, such as the convergence of opposite electrodes.

Dielectric elastomers also have other advantages over air-gap electrostatic actuators, even though both are based on electrostatic force. Several polymers have been identified with breakdown strengths of $300 \mathrm{MV} / \mathrm{m}$ or more in thin films, but breakdown strengths this high are difficult to achieve consistently in air-gap electrostatic devices.

Although the pressure response of dielectric elastomers, as given by Equation 1, is easy to derive, the strain response can be highly nonlinear. For small strains, where an unloaded (free boundary conditions) polymer with an elastic modulus $Y$ is used, the thickness strain $s_{z}$ is given by

$$
s_{z}=-p / Y=-\varepsilon \varepsilon_{0} E^{2} / Y
$$


Equation 2 is useful for qualitative understanding, but in practice its applicability is often limited. In particular, it is limited to free boundary conditions and, more importantly, it is limited to constant $Y$. Dielectric elastomers have recently demonstrated thickness strains exceeding $-75 \%$ and linear planar strains over $300 \%$ in some cases, and $Y$ usually changes significantly at much smaller strains.

Some progress has been made in modeling nonlinear strains in dielectric elastomers by the use of finite element modeling with nonlinear Mooney-Rivlin material properties (6), but in many respects this remains an area of active research.

Most elastomers have a bulk modulus that is orders of magnitude greater than their elastic modulus, so a good approximation is to assume that the volume of the material is unchanged. In this case, one can relate the thickness strain to the planar strains $s_{x}$ and $s_{y}$ as

$$
\left(1+s_{x}\right)\left(1+s_{y}\right)\left(1+s_{z}\right)=1
$$

Thus, if the material is loaded the same in the $\mathrm{x}$ and $\mathrm{y}$ planar directions so that $s_{x}=s_{y}$, one can use Equation 3 to relate the thickness and planar strains. Equation 3 is a good approximation even for very large strains, so it can be used with direct measurements of $s_{x}$ and $s_{y}$ to determine $s_{z}$ even at very high strains.

\section{Experimental Measurements}

The strains in dielectric elastomers are typically very large (3$300 \%$ ), and in the planar direction they are easy to measure optically. This observation led to the use of stretched films with optical measurements as a preferred technique for measuring fundamental performance.

Figure 3 illustrates the experimental setup. A polymer is stretched on a rigid frame. An active area is patterned with compliant electrodes. Typically, a circular pattern is used (biaxial strain test), but line patterns are sometimes preferred when the stretching or prestrain is not isotropic (uniaxial strain test).

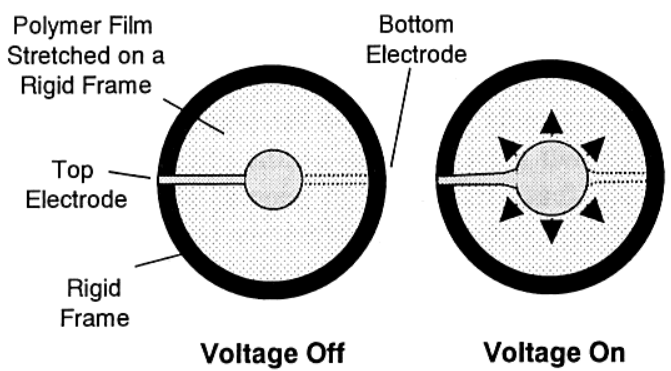

Fig. 3. Setup for measuring strain.

Since the film is prestrained on the frame, absolute strain is of limited interest with regard to its actuation performance. Rather, we are interested in the film's relative strain resulting from the application of the voltage. The relative strain is measured from the voltage-off but prestrained dimensions.

Relative strain is measured optically by means of an automated optical measuring system or a microscope for small samples, or visually with a ruler (for larger, coarser measurements).
Actuation pressure is also an important quantity for actuator materials. Actuation pressure can be measured directly with a force gauge if the film's dimensions are known. Alternatively, Equation 1 can be used to estimate the actuation pressure, the known applied voltage, the measured dielectric constant, and the film's geometry, taking into account any actuated strain. Although the Equation 1 method is less direct than the use of force gauge, the two methods have been shown to yield results in reasonable agreement for the types of polymers of interest (7).

The other actuator parameters of interest are speed of response and theoretical efficiency. The speed of response can be estimated by measuring the magnitude of the strain response as a function of the driving frequency (8). Theoretical efficiency is defined as the efficiency of the material, under the assumptions of ideal electronics and ideal loading. The loss mechanisms of dielectric elastomers are electrical leakage through the film, and viscoelastic mechanical losses. Electrical leakage can be measured with a suitable measurement of leakage current, or estimated if the film resistivity is known. Viscoelastic losses can be measured with dynamic mechanical analyzers designed to measure polymer mechanical moduli and losses under various conditions of loading and strain.

\section{Performance Results}

Table 1 gives the performance results for a silicone dielectric elastomer (9) compared to the final goals. All performance goals were exceeded, and for some parameters (speed of response, in particular) the listed number reflects experimental limitations rather than ultimate limits. The performance described in Table 1 is comparable to or better than the performance parameters of natural muscle (10); hence, the term "artificial muscle" is an appropriate description of the dielectric elastomer technology. Figure 1 graphically shows the comparison with the stress and strain of natural muscle.

Table 1. Experimental results compared with project goals.

\begin{tabular}{|l|c|c|}
\hline Parameter & Goal & Result \\
\hline Strain (\%) & 45 & 63 \\
\hline $\begin{array}{l}\text { Pressure/Density } \\
\left(\mathrm{kPa}-\mathrm{m}^{3} / \mathrm{kg}\right)\end{array}$ & 0.5 & 0.8 \\
\hline $\begin{array}{l}\text { Theoretical } \\
\text { Efficiency (\%) }\end{array}$ & 80 & 85 \\
\hline $\begin{array}{l}\text { Response Time } \\
(\mathrm{ms})\end{array}$ & 0.5 & 0.4 \\
\hline
\end{tabular}

In many respects, the results shown in Table 1 underestimate the full potential of the approach because they focus on one material with respect to a given set of performance goals. Some materials, such as acrylic elastomers, have extraordinary strain (over 300\%), but suffer from viscoelastic losses that slow their response times. Figure 4 shows the very large strains that are feasible (11). The speeds of silicones are over $1 \mathrm{kHz}$, while those of acrylic elastomers are more typically 1-30 Hz. While the speeds of acrylics are lower, their strains and energy densities (mechanical energy output per unit volume or mass) are very large; hence, for applications where high speed is not important, acrylic elastomer may be the best choice. 


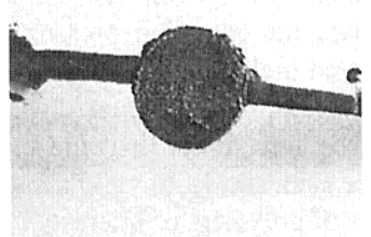

Voltage Off
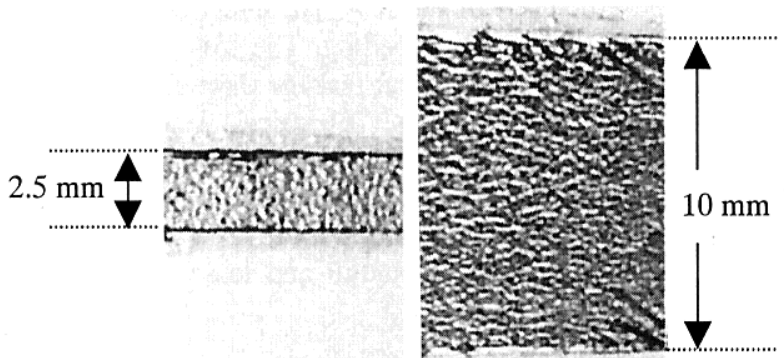

Voltage Off

Voltage On

Fig. 4. Large strains from acrylic elastomers: top photos show biaxial test; bottom photos show uniaxial test yielding $300 \%$ linear strain.

The speed versus strain tradeoffs between silicones and acrylic elastomers illustrate an advantage of the dielectric elastomer approach. There are a huge range of polymers and many variations within classes of polymers. Virtually all insulating polymers show some Maxwell stress response, and the wealth of candidate materials suggests that a dielectric elastomer can be tailored to the requirements of the specific application. While many competitive materials can also be modified, dielectric elastomers are perhaps the broadest class of actuator materials and should therefore be among the most flexible in material design.

\section{Fabrication}

Dielectric elastomers can be fabricated in a variety of ways. The polymer films themselves can be cast, sprayed, or spun. Dip coating can be used to make complex shapes. All of these polymer fabrication methods have been used to make dielectric elastomer actuator materials. The optimal choice of fabrication method depends on the application as well as on cost and performance tradeoffs.

Spin coating generally makes the most uniform, highestquality polymer films. It can also be used to make the thinnest films: spin coating has been used to make good-quality silicone films as thin as $1 \mu \mathrm{m}$. Spin coating is also desirable when an artificial muscle film is to be made in situ on a permanent substrate such as silicon. This technique has been used to fabricate arrays of diaphragm actuators.
While spin coating yields the highest-quality film, other polymer fabrication methods can be better for some applications. Flat casting is perhaps the lowest-cost method of fabrication. Spray coating may be useful for low-cost, large structures where performance is not critical. Dip coating is attractive for applications requiring a complex shape either as a permanent part of the dipped structure or as a released shape.

Electrode materials are more limited than polymers, but several good electrode materials and associated fabrication techniques have been demonstrated. The critical requirement for the electrode material is that it be compliant. Ideally, it should also be smooth, reasonably conductive in very thin films, and environmentally tolerant. It must also be chemically compatible with the intended polymer material.

Some of the best compliant-electrode materials are based on carbon particles in the form of graphite, carbon black, carbon nanotubes, and carbon fibrils. Carbon particles can be mixed in polymer binders to make a compliant electrode. Ionic conductors, such as water-based gels with salts, can also be used as compliant electrodes, but in general ions are problematic with dielectric elastomers because ion migration into the film causes premature failure.

Metal conductors can also be used with dielectric elastomers. Uniform metal electrodes typically fracture at around $2-4 \%$ strain, so techniques must be used that allow higher strains. The metal can be patterned to bend rather than directly elongate. An example of this approach is illustrated by zig-zag gold traces microfabricated on silicone (6). Such structures can strain up to $80 \%$ without losing conductivity. More research is needed in this area, but the results to date indicate that metals can be used to make at least some dielectric elastomer electrodes. Such patterned metal conductors may be desirable with microactuators because they can be patterned with high precision.

\section{Applications and Proof-of-Principle Devices}

It is important that a new actuator technology be shown to be configurable into useful actuator designs. Dielectric elastomer artificial muscle can be configured into a wide range of actuator configurations. Many of these types of actuator are analogues of other existing types, while others are new. We demonstrated a variety of actuators using dielectric elastomers to show that this new technology could be designed for practical applications. To this end, we built these actuators on the proof-of-principle level to show their functionality but in general we did not optimize them for specific performance. Here we describe a few of the types of actuators that we built, and discuss their important features.

Framed Actuators. The simplest actuators are formed by stretching a dielectric elastomer film on a frame. The strain test photos in Figure 4 illustrate the type of actuation that can be achieved with this design. Multiple layers can be added to increase actuation force. The framed actuator can be used directly for applications such as light modulation. Figure 5 shows a framed actuator exhibiting an interesting optical effect: when activated, the film changes from dark to semitransparent. Alternatively, rigid mechanical coupling can be included to provide a conventional mechanical output analogous to that of an electromagnetic solenoid but with much higher actuation performance. 


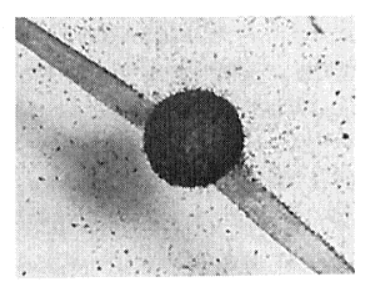

Voltage Off

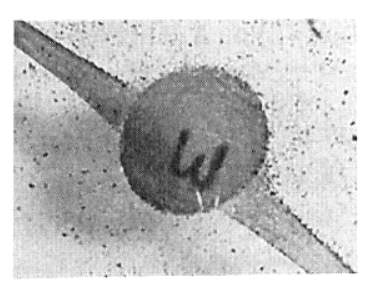

Voltage On

Fig. 5. Optical effect of eactivating a stretched film actuator.

Diaphragm Actuators. Dielectric elastomers are ideal for diaphragm actuators for pumps, speakers, and other applications requiring the movement of fluids. As with stretched film actuators, rigid mechanical coupling can also be used with some designs to provide conventional mechanical output.

We have demonstrated a wide range of diaphragm actuators. Diaphragms as small as $50 \mu \mathrm{m}$ and as large as $10 \mathrm{~cm}$ have been successfully actuated, and diaphragm arrays have also been built on substrates such as plastic and silicon.

Possible diaphragm pressures have not been extensively explored, but pressures of $20 \mathrm{kPa}$ have been demonstrated. Theoretical calculations indicate that much higher diaphragm pressures should be feasible, up to around $1 \mathrm{MPa}$.

Strokes are relatively large compared to those produced with other types of diaphragms, such as piezoelectric-driven diaphragms. Typically, silicone dielectric elastomers achieved displacements of $10 \%$ of the diaphragm diameter, while acrylic elastomers could easily achieve displacements of $50 \%$ of the diaphragm diameter. These acrylic elastomers can actuate from an essentially flat configuration to a hemispherical one.

These large displacements illustrate an attractive feature of dielectric elastomer diaphragms, their large volume displacements per stroke.

We demonstrated the potential of diaphragm actuator for micropump applications by building a simple proof-of-principle minipump. The $20 \mathrm{~mm}$ diaphragm could pump up to $40 \mathrm{ml} / \mathrm{min}$ of air at pressures of about $1 \mathrm{kPa}$.

Diaphragm actuators can also operate using lower-voltage films. Dielectric elastomer voltages depend on a number of parameters such as film thickness, dielectric constant, and elastic modulus. Because dielectric elastomers are high-performance electrostatic actuators, their voltages tend to be high relative to those of electromagnetic devices. Ongoing research is seeking to reduce the operating voltage, but for now practical MEMS devices are limited to $100-300 \mathrm{~V}$ or higher for full strain performance (lower voltages can be used for less than $5 \%$ strains). We have built diaphragm actuators with good strain performance at these voltages. To illustrate their potential, we coupled a mechanical structure to a $5-\mathrm{mm}$ diaphragm and configured it with a reflective surface as a micro light scanner. Operating voltages were typically 190-300 V.

Unimorph and Bimorph Actuators. We also built micro light scanners using unimorph dielectric elastomer actuators. Unimorphs are formed by bonding an active dielectric elastomer to a thin beam that can bend but not stretch. The elongation of the dielectric elastomer causes the beam to bend. Bimorph actuators are similar, except that active film is used on both sides of the beam to enhance the bending.
Unimorph and bimorph actuators based on dielectric elastomers are similar in a number of ways to piezoelectric unimorphs and bimorphs. However, dielectric elastomer unimorphs and bimorphs can bend in much sharper angles because the strains they achieve are orders of magnitude larger. Bending angles as high as 270 degrees have been observed, and 360 -degree or more bending is undoubtedly possible with optimization. Beam lengths were typically 2 to $10 \mathrm{~mm}$, and operating frequencies of $10-50 \mathrm{~Hz}$ were achieved.

Most of our work was devoted to unimorph actuators. Arrays of 5-10 unimorphs were demonstrated: these can be fabricated in batch processes. We demonstrated arrays of unimorphs pushing paper, which suggests paper-feeder applications. Unimorphs can also be used to change the angle of mirrors; Figure 6 shows laser light scanning with a $2-\mathrm{mm}$ unimorph.

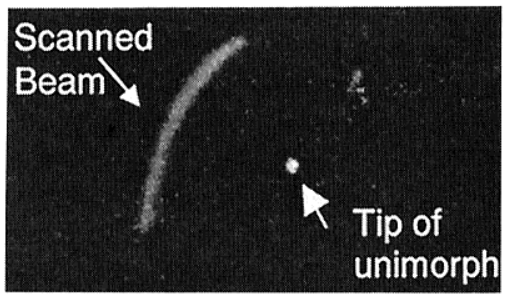

Fig. 6. Micro light scanning with a unimorph.

Rolled Actuators. Dielectric elastomer film can be rolled to form a compact actuator. (These rolled actuators were built in 1995 before more recent advances in the technology.) We demonstrated roll actuators measuring approximately $10 \mathrm{~mm}$ in length and $3 \mathrm{~mm}$ in diameter and providing $0.5-1 \mathrm{~mm}$ displacement and $0.01-0.02 \mathrm{~N}$ force (1-2 $\mathrm{g}$ force).

These rolled actuators can be used for general-purpose small actuators. We also showed that they could be used as the primary actuator for inchworm-type robots. Electrostatic clamps were placed at each end of a rolled actuator, and the clamps and roll actuation were phased to controllably move the device forward and backward. The artificial muscle inchworm-type robot could climb vertically and had speeds up to $40 \mathrm{~mm} / \mathrm{s}$.

\section{Conclusion}

Dielectric elastomers show promise as a new actuation technology with natural muscle-like performance. They achieve strains of over $300 \%$, among the highest ever reported for fieldactutated materials. Their pressures are attractive at 1-8 MPa. Response times and theoretical efficiencies depend on the particular polymer and operating conditions, but response times of $0.4 \mathrm{~ms}$ and theoretical efficiencies of $85 \%$ have been shown.

We have developed fabrication methods for this new technology and have explored a wide range of materials with promising results. We also built a number of proof-of-principle actuators and successfully demonstrated them to show the feasibility of using them to address practical applications. 
Although much progress has been made on the fundamental technology, many technical challenges remain to reach practical applications. Electronics for driving the relatively high voltages of the dielectric elastomer, though feasible, are not currently available and must be developed. Many aspects of lifetime and environmental tolerance also need to be carefully examined, to enable the technology to succeed.

These technical challenges are typical of most new technology, so in spite of the remaining challenges, dielectric elastomer artificial muscle is a promising new technology that may be able to address many new and old applications on both micro and macro scales.

(Manuscript received July. 10, 2001)

\section{References}

(1) R. Pelrine, J. Eckerle, and S. Chiba, "Review of Artificial Muscle Approaches," (invited), Proc. Third International Symposium on Micro Machine and Human Science, Nagoya, Japan, pp. 1-9 October 1992.

(2) R.J. Full, "Invertebrate locomotor systems," in The Handbook of Comparative Physiology, ed. W. Danzler, Oxford University Press, pp. 853-930, 1997.

(3) M. Zhenyi, J.I. Scheinbeim, J.W. Lee, and B.A. Newman, "High Field Electrostrictive Response of Polymers," $J$. Polymer Sciences, Part B-Polymer Physics 32, pp. 27212731, 1994.

(4) G. Kofod, R. Kornbluh, R. Pelrine, and P. Sommer-Larsen, "Actuation response of polyacrylate dielectric elastomers," to be published in Proc. SPIE Vol. 4329 (Electroactive Polymer Actuators and Devices, 2001) from Smart Structures and Materials Symposium 2001, Newport Beach, California, 4-8 March 2001.

(5) R. Pelrine, R. Kornbluh, J. Joseph, and J. Marlow, "Analysis of the Electrostriction of Polymer Dielectrics with Compliant Electrodes as a Means of Actuation," Sensors and Actuators A: Physical 64, pp. 77-85, 1998.

(6) Kornbluh, R., R. Pelrine, J. Joseph, R. Heydt, Q. Pei, and S. Chiba, "High-Field Electrostriction of Elastomeric Polymer Dielectrics for Actuation," Proc. SPIE International Symposium on Smart Structures and Materials: ElectroActive Polymer Actuators and Devices, pp. 149-161, Newport Beach, California (March 1999).

(7) R. Pelrine, R. Kornbluh, and G. Kofod, "High-Strain Actuator Materials Based on Dielectric Elastomers," Advanced Materials 2000 12:16, pp. 1223-1225, 2000.

(8) R. Kornbluh, R. Pelrine, Q. Pei, S. Oh, and J. Joseph, "Ultrahigh Strain Response of Field-Actuated Elastomeric Polymers," Proc. SPIE, Smart Structures and Materials 2000: Electroactive Polymer Actuators and Devices (EAPAD), Y. Bar-Cohen, ed., 3987, pp. 51-64, 2000.
(9) R. Pelrine, P. Sommer-Larsen, R. Kornbluh, R. Heydt, G. Kofod, Q. Pei, and P. Gravesen, "Applications of Dielectric Elastomer Actuators," to be published in Proceedings of SPIE Vol. 4329 (Electroactive Polymer Actuators and Devices, 2001) from Smart Structures and Materials Symposium 2001, March 4-8, Newport Beach, California, 4-8 March 2001.

(10) R. Full and K. Meijer, "Chapter 3.0-Natural Muscles as an Electromechanical System," in Electroactive Polymer (EAP) Actuators as Artificial Muscles-Reality, Potential and Challenges, SPIE Press, Y. Bar-Cohen, ed., pp. 67-83.

(11) R. Pelrine, R. Kornbluh, Q. Pei, and J. Joseph, "High-Speed Electrically Actuated Elastomers with Over 100\% Strain," Science 287:5454, pp. 836-839, 2000.

Ron Pelrine (non-member) received a Ph.D. in mechanical Engineering at the University of Texas at Austin and is presently a program director at SRI International. He has worked on magnetic micro devices, inkjets,levitation, robots, sensors, and advanced actuators

Roy Kornbluh (non-member) received his Masters degree in

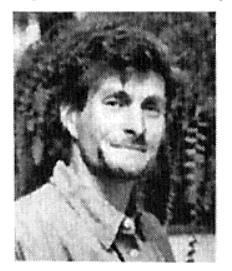
mechanical engineering from the Massachusetts Institute of Technology and his bachelors in mechanical engineering from Cornell University. Roy Kornbluh is a Senior Research Engineer at SRI International, where he has been involved with projects relating to the development of electromechanical devices. For the past six years, Roy has focused on the development of electroactive polymer transducers.

Seiki Chiba (Member) received a Ph.D. in Metallurgy \&

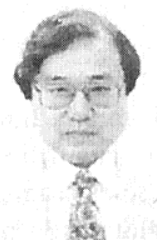

Material Science at the University of Wales (Britain) and is presently an executive director at SRI International. He has been supervising advanced R\&D projects awarded from Japanese Government. His email :schiba@sri.co.jp 